Internet Engineering Task Force (IETF)

E. Lear, Ed.

Request for Comments: 7979

R. Housley, Ed.

Category: Informational

August 2016

ISSN : $2070-1721$

Response to the IANA Stewardship Transition Coordination Group (ICG)

Request for Proposals on the IANA Protocol Parameters Registries

Abstract

The U.S. National Telecommunications and Information Administration

(NTIA) solicited a request from the Internet Corporation for Assigned Names and Numbers (ICANN) to propose how the NTIA should end its oversight of the Internet Assigned Numbers Authority (IANA)

functions. After broad consultations, ICANN in turn created the IANA Stewardship Transition Coordination Group. That group solicited proposals for the three major IANA functions: names, numbers, and protocol parameters. This document contains the IETF response to that solicitation for protocol parameters. It was included in an aggregate response to the NTIA alongside those for names and numbering resources that are being developed by their respective operational communities. A reference to that response may be found in the introduction, and additional correspondence is included in the Appendix.

Status of This Memo

This document is not an Internet Standards Track specification; it is published for informational purposes.

This document is a product of the Internet Engineering Task Force (IETF). It represents the consensus of the IETF community. It has received public review and has been approved for publication by the Internet Engineering Steering Group (IESG). Not all documents approved by the IESG are a candidate for any level of Internet Standard; see section 2 of RFC 7841.

Information about the current status of this document, any errata, and how to provide feedback on it may be obtained at http://www.rfc-editor.org/info/rfc7979. 
Copyright Notice

Copyright (c) 2016 IETF Trust and the persons identified as the document authors. All rights reserved.

This document is subject to BCP 78 and the IETF Trust's Legal Provisions Relating to IETF Documents (http://trustee.ietf.org/license-info) in effect on the date of publication of this document. Please review these documents carefully, as they describe your rights and restrictions with respect to this document. Code Components extracted from this document must include Simplified BSD License text as described in section $4 . e$ of the Trust Legal Provisions and are provided without warranty as described in the Simplified BSD License.

Table of Contents

1. IETF Introduction . . . . . . . . . . . . . . . . . . . . . . 3

2. The Formal RFP Response. . . . . . . . . . . . . . . . . . 4

3. IANA Considerations . . . . . . . . . . . . . . . . . . 20

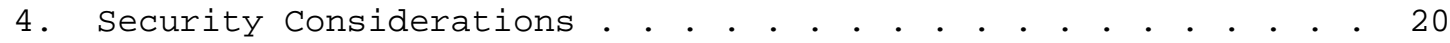

5. IAB Note . . . . . . . . . . . . . . . . . . . . . 20

6. Acknowledgments . . . . . . . . . . . . . . . . . 20

7. References . . . . . . . . . . . . . . . . . . . . . . 20

7.1. Normative References . . . . . . . . . . . . . . . 20

7.2. Informative References . . . . . . . . . . . . . . . . 22

Appendix A. The Charter of the IANA Stewardship Coordination Group (ICG) . . . . . . . . . . . . . . . 25

Appendix B. IANA Stewardship Transition Coordination Group Request for Proposals • • • • • • • • • • • . 28

Appendix C. Correspondence of the IETF to the ICG . . . . . . 34 Authors' Addresses . . . . . . . . . . . . . . . . . . 37 


\section{IETF Introduction}

In March of 2014, the U.S. National Telecommunications and Information Administration (NTIA) announced its intent to transition oversight of Internet Assigned Numbers Authority (IANA) functions [NTIA-Announce]. In that announcement, NTIA asked the Internet Corporation for Assigned Names and Numbers (ICANN) to establish a process to deliver a proposal for transition. As part of that process, the IANA Stewardship Transition Coordination Group (ICG) was formed. The charter for the ICG can be found in Appendix A. The ICG in turn solicited proposals regarding post-transition arrangements from the names, numbers, and protocol parameters communities in order to put forth a proposal to the NTIA. The final request for proposal (RFP) can be found in Appendix B. The response from the ICG to the NTIA may be found at [ICG-Response].

While there are interactions between all of the IANA functions and IETF standards, this document specifically addresses the protocol parameters registries function. Section 1 (this section) contains an introduction that is sourced solely within the IETF. Section 2 contains the questionnaire that was written by the ICG and a formal response by the IETF. We have quoted questions from that questionnaire with ">>> ", and we have prefaced answers to questions being asked with "IETF Response:". Note that there are small changes to the questions asked in order to match the RFC format.

We note that the following text was stated as a footnote in the original RFP:

In this RFP, "IANA" refers to the functions currently specified in the agreement between NTIA and ICANN [http://www.ntia.doc.gov/page/iana-functions-purchase-order] as well as any other functions traditionally performed by the IANA functions operator. SAC-067 [https://wWW.icann.org/en/system/files/files/sac-067-en.pdf] provides one description of the many different meanings of the term "IANA" and may be useful reading in addition to the documents constituting the agreement itself. 


\section{The Formal RFP Response}

The entire Request for Proposals, including introduction, can be found in Appendix B.

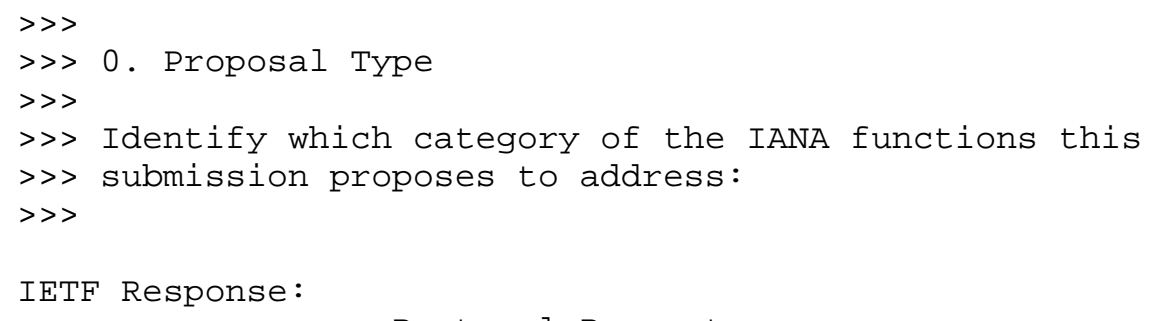

Many IETF protocols make use of commonly defined protocol parameters. These parameters are used by implementers, who are the primary users of the IETF standards and other documents. To ensure consistent interpretation of these parameter values by independent implementations, and to promote universal interoperability, these IETF protocol specifications define and require globally available registries containing the parameter values and a pointer to any associated documentation. The IETF uses the IANA protocol parameters registries to store this information in a public location. The IETF community presently accesses the protocol parameter registries via references based on the iana.org domain name, and makes use of the term "IANA" in the protocol parameter registry processes [RFC5226].

ICANN currently operates the. ARPA top level domain on behalf of the Internet Architecture Board (IAB). This zone is used for certain Internet infrastructure services that are delegated beneath it. The IETF considers. ARPA part of the protocol parameters registries for purposes of this response. 


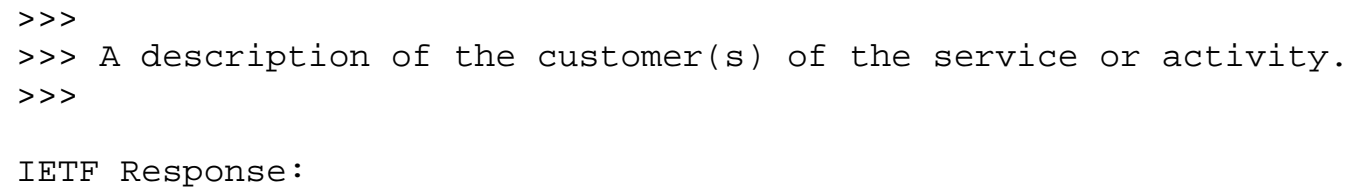

The IETF is a global organization that produces voluntary standards, whose mission is to produce high quality, relevant technical and engineering documents that influence the way people design, use, and manage the Internet in such a way as to make the Internet work better [RFC3935]. IETF standards are published in the RFC series. The IETF is responsible for the key standards that are used on the Internet today, including IP, TCP, DNS, BGP, and HTTP, to name but a few.

The IETF operates in an open and transparent manner [RFC6852]. The processes that govern the IETF are also published in the RFC series. The Internet Standards Process is documented in [RFC2026]. That document explains not only how standards are developed, but also how disputes about decisions are resolved. RFC 2026 has been amended a number of times [BCP9info]. The standards process can be amended in the same manner that standards are approved. That is, someone proposes a change by submitting a temporary document known as an Internet-Draft, the community discusses it, and if rough consensus can be found the change is approved by the Internet Engineering Steering Group (IESG), who also have day-to-day responsibility for declaring IETF consensus on technical decisions, including those that affect the IANA protocol parameters registries. Anyone may propose a change during a Last Call, and anyone may participate in the community discussion. 


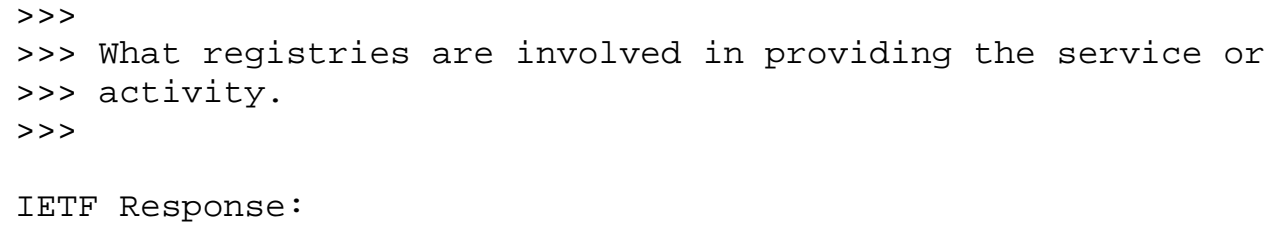

- The IETF has specified a number of special use registries with regard to domain names. These registries require coordination with ICANN as the policy authority for the DNS root, including community groups that are responsible for ICANN policy on domain names such as the Generic Names Supporting Organization (GNSO) and the Country Code Names Supporting Organization (ccNSO). There are 
already mechanisms in place to perform this coordination, and the capacity to modify those mechanisms to meet new conditions as they might arise. [RFC6761]

- The IETF specifies the DNS protocol. From time to time there have been and will be updates to that protocol. As we make changes we will broadly consult the operational community about the impact of those changes, as we have done in the past.

- The IETF specifies minimum requirements for root servers. [RFC2870] Those requirements are currently under review, in consultations with the root server community.

- The routing architecture has evolved over time, and is expected to continue to do so. Such evolution may have an impact on appropriate IP address allocation strategies. If and when that happens, the IETF will consult and coordinate with the RIR community, as we have done in the past.

- The IETF is responsible for policy relating to the entire IP address space and AS number space. Through the IANA protocol parameters registries, the IETF delegates unicast IP address and AS number ranges to the RIRs [RFC7020], [RFC7249]. Special address allocation, such as multicast and anycast addresses, often require coordination. Another example of IP addresses that are not administered by the RIR system is Unique Local Addresses (ULAs) [RFC4193], where local networks employ a prefix that is not intended to be routed on the public Internet. New special address allocations are added, from time to time, related to the evolution of the standards. In all cases, these special assignments are listed in the IANA protocol paramters registries.

- The IETF maintains sub-registries for special IPv4 and IPv6 assignments. These are specified in [RFC3307], [RFC5771], and [RFC6890]. The IETF coordinates such assignments with the RIRs.

- Changes to IETF standards may have impact on operations of RIRS and service providers. A recent example is the extensions to BGP to carry the Autonomous system numbers as four-octet entities [RFC6793]. It is important to note that this change occurred out of operational necessity, and it demonstrated strong alignment between the RIRs and the IETF. 


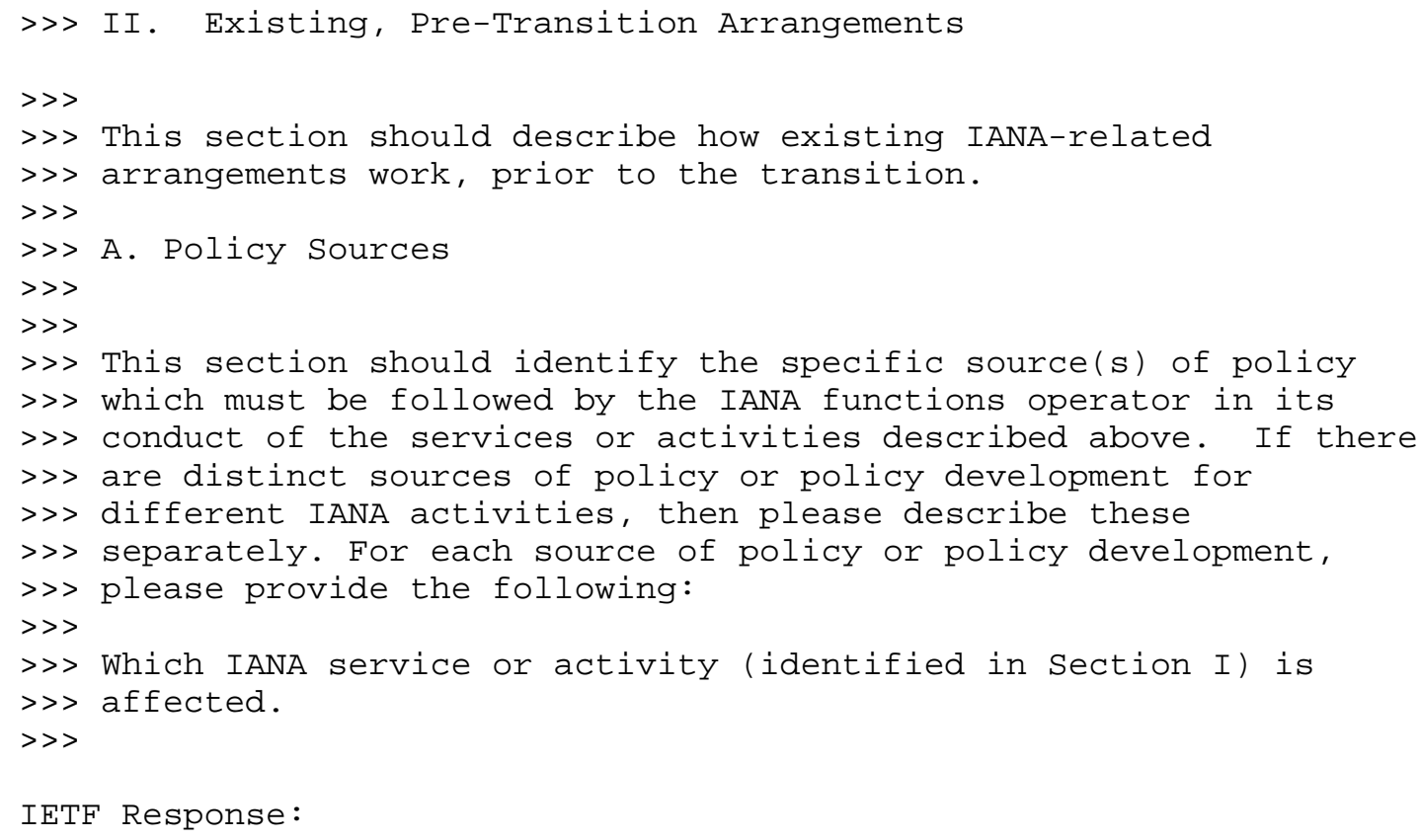




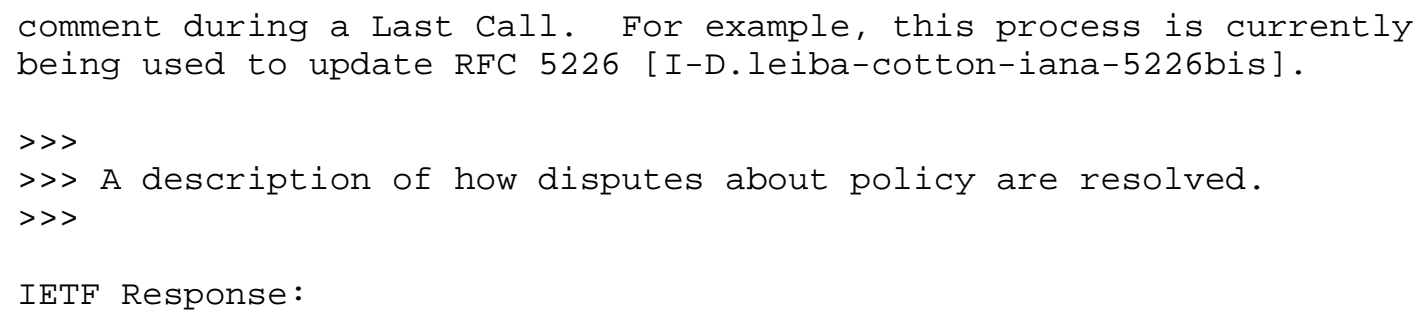


IETF Response:

The protocol parameters registries.

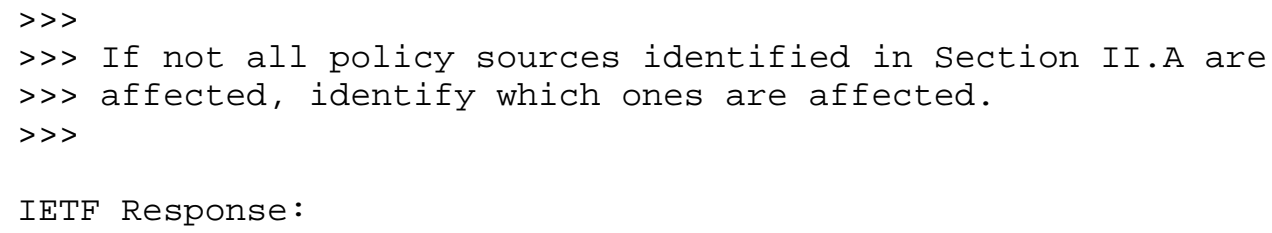

The Internet Architecture Board (IAB) is an oversight body of the IETF whose responsibilities include, among other things, confirming appointment of IESG members, managing appeals as discussed above, management of certain domains, including. ARPA [RFC3172], and general architectural guidance to the broader community. The IAB must approve the appointment of an organization to act as IANA operator on behalf of the IETF. The IAB is also responsible for establishing liaison relationships with other organizations on behalf of the IETF. The IAB's charter is to be found in [RFC2850].

The IAB members are selected and may be recalled through a Nominating Committee (NOMCOM) process, which is described in [RFC3777] and its updates. This process provides for selection of active members of the community who themselves agree upon a slate of candidates. The active members are chosen randomly from volunteers with a history of participation in the IETF, with limits regarding having too many active members with the same affiliation. The selection of the active members is performed in a manner that makes it possible for anyone to verify that the correct procedure was followed. The slate of candidates selected by the active members are sent to the Internet Society Board of Trustees for confirmation. In general, members are appointed for terms of two years. The IAB selects its own chair.

The IAB provides oversight of the protocol parameters registries of the IETF, and is responsible for selecting appropriate operator(s) and related per-registry arrangements. Especially when relationships 
among protocols call for it, registries are at times operated by, or in conjunction with, other bodies. Unless the IAB or IETF has concluded that special treatment is needed, the operator for registries is currently ICANN.

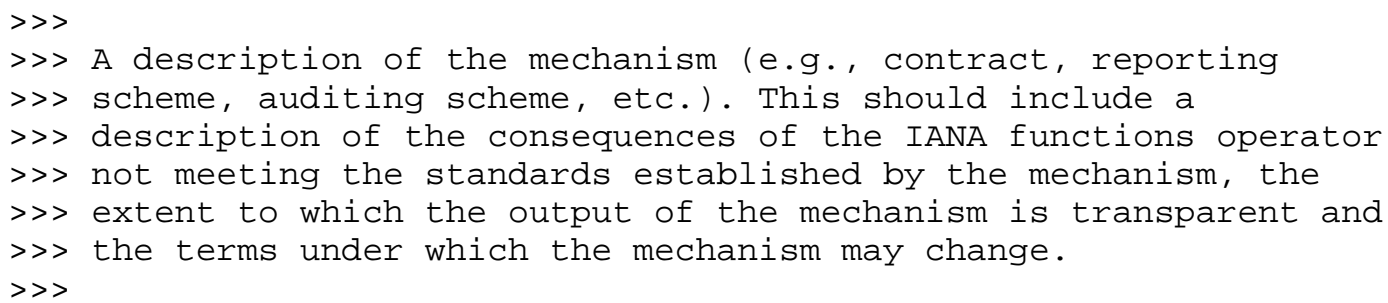




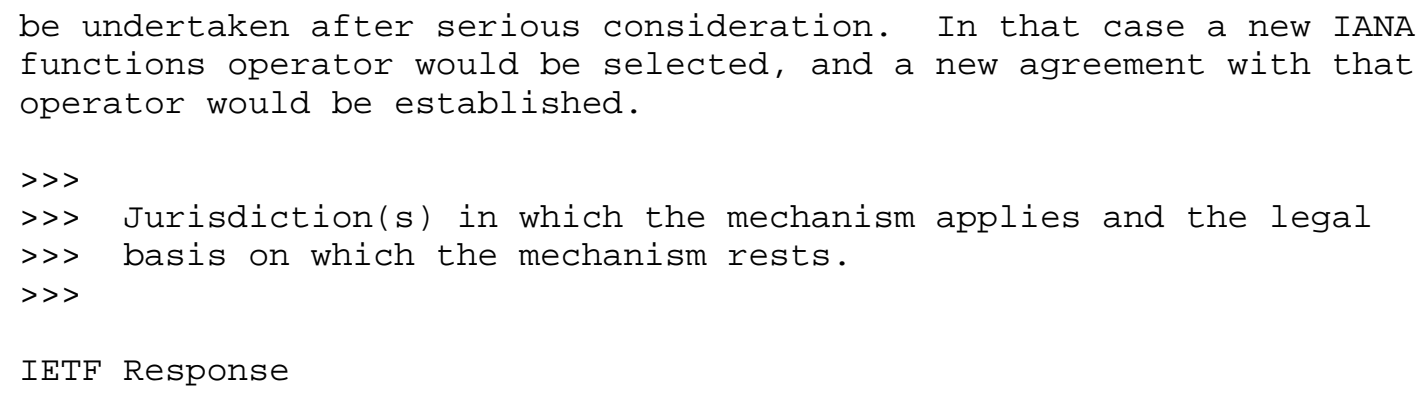


ICANN. RFC 2860 remains in force and has served the IETF community very well. RFC 6220 has laid out an appropriate service description and requirements.

However in the absence of the NTIA contract a few new arrangements may be needed in order to ensure the IETF community's expectations are met. Those expectations are the following:

- The protocol parameters registries are in the public domain. It is the preference of the IETF community that all relevant parties acknowledge that fact as part of the transition.

- It is possible in the future that the operation of the protocol parameters registries may be transitioned from ICANN to subsequent operator(s). It is the preference of the IETF community that, as part of the NTIA transition, ICANN acknowledge that it will carry out the obligations established under C.7.3 and I.61 of the current IANA functions contract between ICANN and the NTIA [NTIA-Contract] to achieve a smooth transition to subsequent operator(s), should the need arise. Furthermore, in the event of a transition it is the expectation of the IETF community that ICANN, the IETF, and subsequent operator(s) will work together to minimize disruption in the use the protocol parameters registries or other resources currently located at iana.org.

In developing our response we have been mindful of the following points that the IETF community has discussed over the last year [ProtoParamEvo14] that have led to the following guiding principles for IAB efforts that impact IANA protocol parameter registries. These principles must be taken together; their order is not significant.

1. The IETF protocol parameters registries function has been and continues to be capably provided by the Internet technical community.

The strength and stability of the function and its foundation within the Internet technical community are both important given how critical protocol parameters are to the proper functioning of IETF protocols.

We think the structures that sustain the protocol parameters registries function need to be strong enough that they can be offered independently by the Internet technical community, without the need for backing from external parties. And we believe we largely are there already, although the system can be strengthened further, and continuous improvements are being made. 
2. The protocol parameters registries function requires openness, transparency, and accountability.

Existing documentation of how the function is administered and overseen is good [RFC2860], [RFC6220]. Further articulation and clarity may be beneficial. It is important that the whole Internet community can understand how the function works, and that the processes for registering parameters and holding those who oversee the protocol parameters function accountable for following those processes are understood by all interested parties. We are committed to making improvements here if necessary.

3. Any contemplated changes to the protocol parameters registries function should respect existing Internet community agreements.

The protocol parameters registries function is working well. The existing Memorandum of Understanding in RFC 2860 defines "the technical work to be carried out by the Internet Assigned Numbers Authority on behalf of the Internet Engineering Task Force and the Internet Research Task Force." Any modifications to the protocol parameters registries function should be made using the IETF process to update RFC 6220 and other relevant RFCs. Put quite simply: evolution, not revolution.

4. The Internet architecture requires and receives capable service by Internet registries.

The stability of the Internet depends on capable provision of not just IETF protocol parameters, but IP numbers, domain names, and other registries. Furthermore, DNS and IPv4/IPv6 are IETF-defined protocols. Thus we expect the role of the IETF in standards development, architectural guidance, and allocation of certain name/ number parameters to continue. IP multicast addresses and specialuse DNS names are two examples where close coordination is needed. The IETF will continue to coordinate with ICANN, the RIRs, and other parties that are mutually invested in the continued smooth operation of the Internet registries. We fully understand the need to work together.

5. The IETF will continue management of the protocol parameter registry function as an integral component of the IETF standards process and the use of resulting protocols.

RFC 6220 specifies the role and function of the protocol parameters registry, which is critical to IETF standards processes and IETF protocols. The IAB, on behalf of the IETF, has the responsibility to define and manage the relationship with the protocol registry operator role. This responsibility includes the selection and 
management of the protocol parameter registry operator, as well as management of the parameter registration process and the guidelines for parameter allocation.

6. The protocol parameters registries are provided as a public service.

Directions for the creation of protocol parameters registries and the policies for subsequent additions and updates are specified in RFCs. The protocol parameters registries are available to everyone, and they are published in a form that allows their contents to be included in other works without further permission. These works include, but are not limited to, implementations of Internet protocols and their associated documentation.

These principles will guide the IAB, IAOC, and the rest of the IETF community as they work with ICANN to establish future IANA performance metrics and operational procedures.

>> IV Transition Implications

$>>>$

$>>$ This section should describe what your community views as the $>>$ implications of the changes it proposed in section III. These >> implications may include some or all of the following, or other $>>$ implications specific to your community:

$>>>$

$>>$ O Description of operational requirements to achieve continuity $>>$ of service and possible new service integration throughout $>>$ the transition.

$>>$ O Risks to operational continuity

$>>$ O Description of any legal framework requirements in the $>>$ absence of the NTIA contract

$>>>$ Description of how you have tested or evaluated the

$>>\quad$ workability of any new technical or operational methods

$>$ proposed in this document and how they compare to established $>$ arrangements.

$>>>$

IETF Response:

No structural changes are required for the handling of protocol parameters. The principles listed above will guide IAB, IAOC, and the rest of the IETF community as they work with ICANN to establish future IANA performance metrics and operational procedures, as they have in the past. 


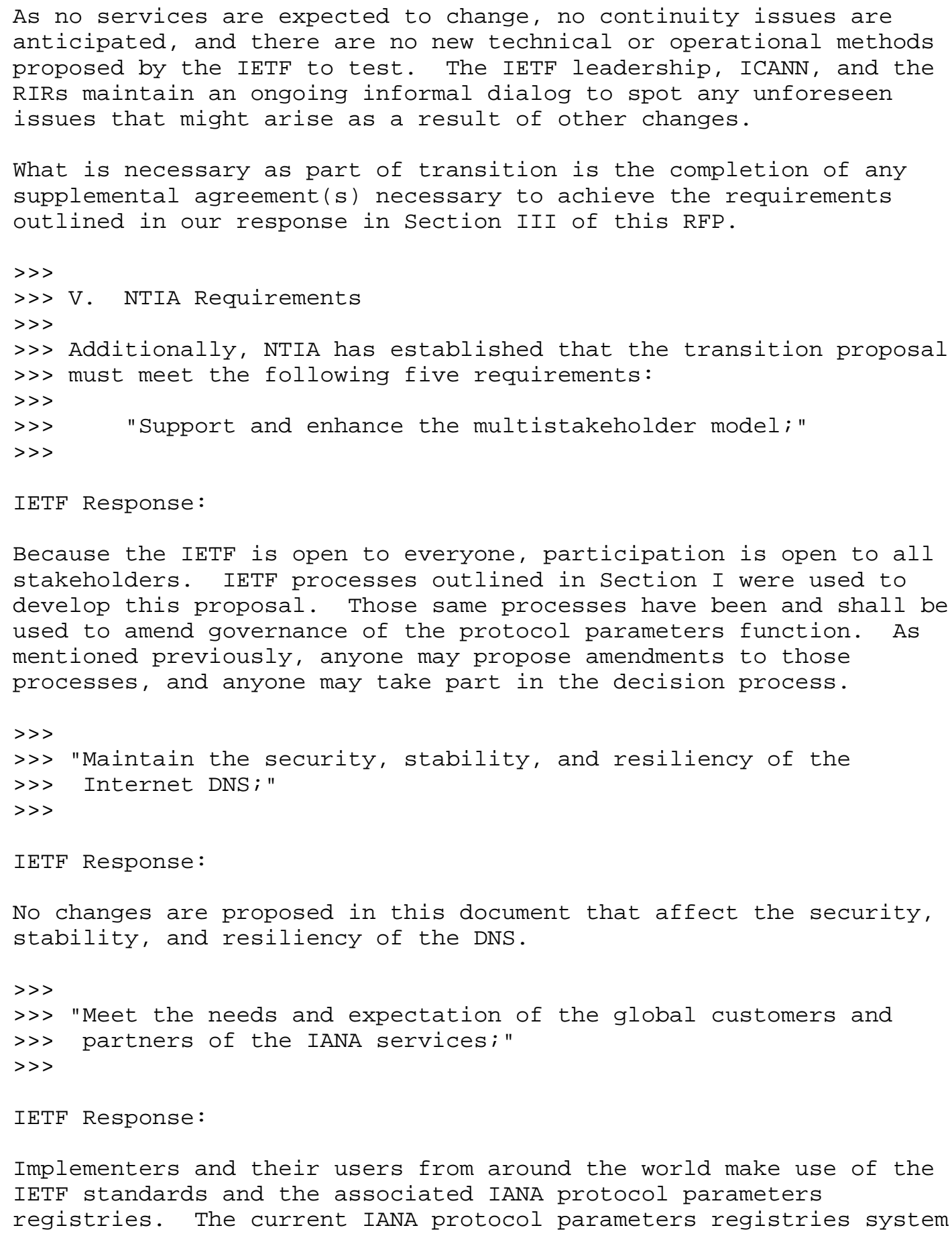




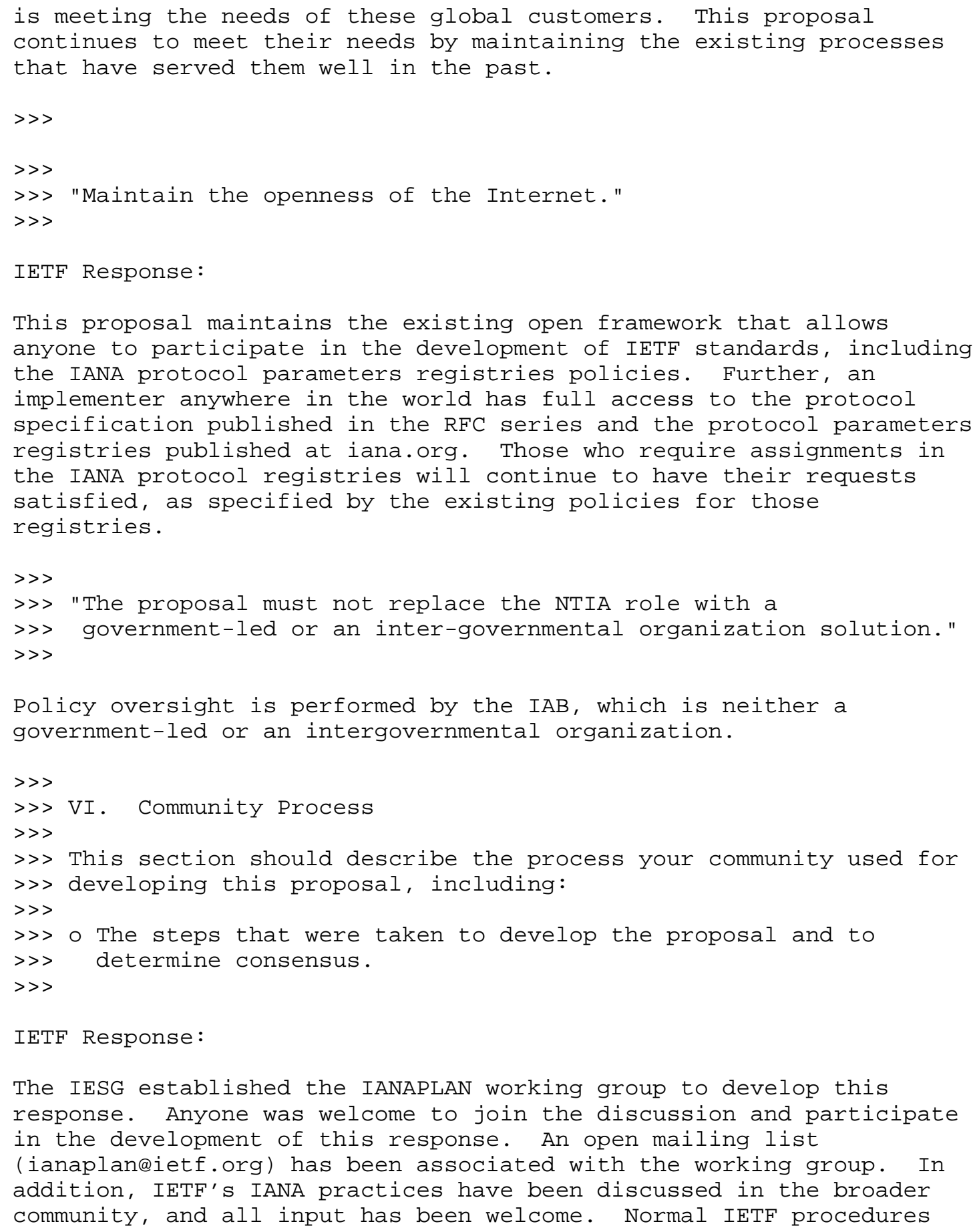


[RFC2026] [RFC2418] were used to determine rough consensus. The chairs of the working group reviewed open issues and, after an internal working group last call, determined that all had been satisfactorily addressed, and subsequently the IESG did a formal IETF-wide Last Call followed by a formal review and determined that the document had rough consensus.

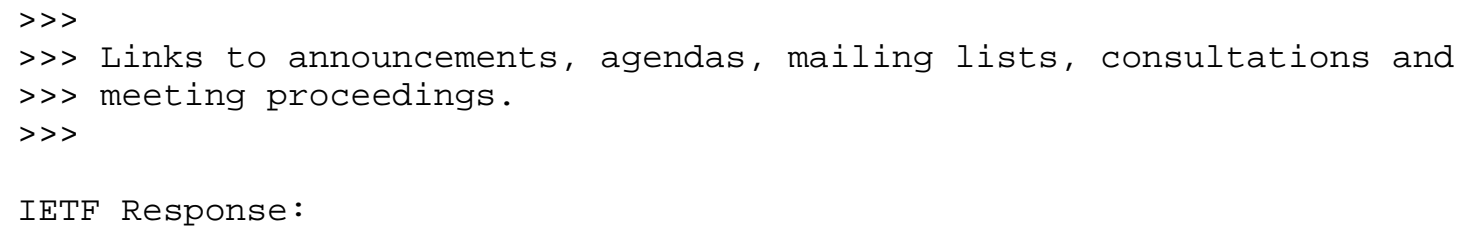

The working group discussion: http://www.ietf.org/mail-archive/web/ianaplan/current/ mailist.html

2014-10-06 Interim Meeting Agenda, Minutes, and presentations: http://www.ietf.org/proceedings/interim/2014/10/06/ianaplan/ proceedings.html

Working group last call: http://mailarchive.ietf.org/arch/msg/ianaplan/ EGF 9 rf Jxn 5QpQnRXmS2QxYKYR8k

Agenda from IETF 91 IANAPLAN WG meeting: http://www.ietf.org/proceedings/91/agenda/agenda-91-ianaplan

Minutes of IETF 91 IANAPLAN WG meeting: http://wWw. ietf.org/proceedings/91/minutes/minutes-91-ianaplan 


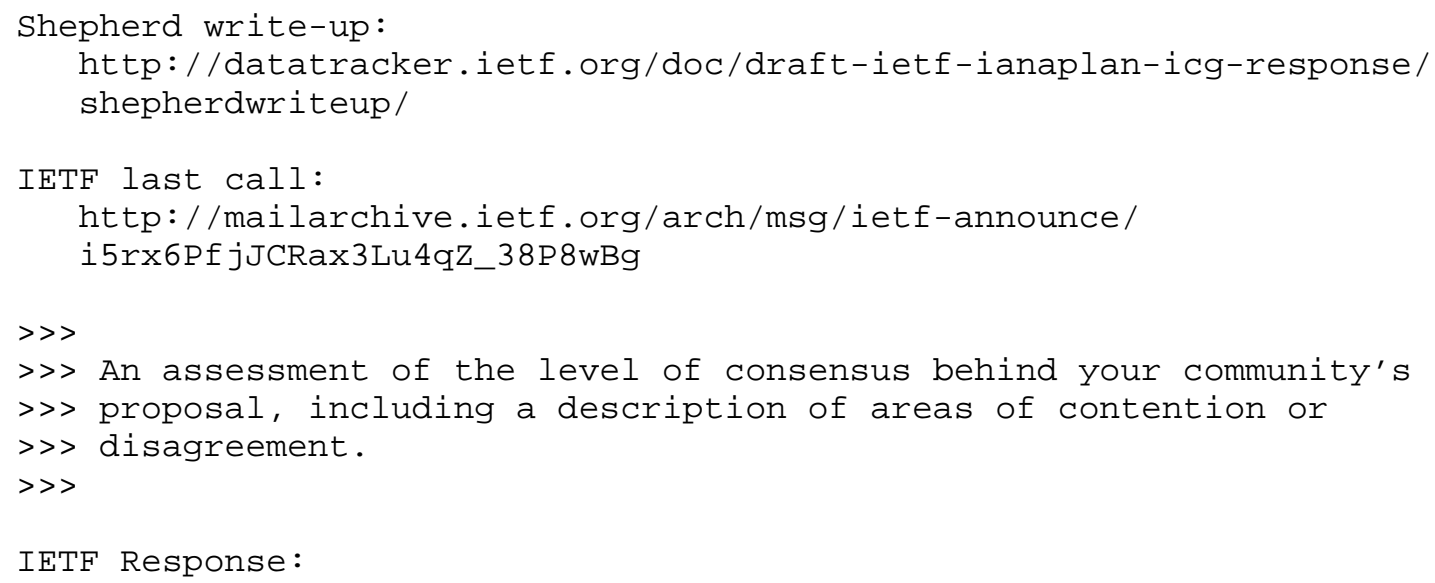


of which resulted in text changes. There was some discussion of comments already discussed earlier in the process, and but no new objections were raised during the IETF last call. A summary of the last call comments can be found from here:

http://www.ietf.org/mail-archive/web/ianaplan/current/msg01500.html

New draft versions were prepared that took into account all the agreed changes from the last call. The final version was then approved by the IESG.

3. IANA Considerations

This memo is a response to a request for proposals. No parameter allocations or changes are sought.

4. Security Considerations

While the agreement, supplements, policies, and procedures around the IANA function have shown strong resiliency, the IETF will continue to work with all relevant parties to facilitate improvements while maintaining availability of the IANA registries.

5. IAB Note

The IAB supports the response in this document.

6. Acknowledgments

This document describes processes that have been developed by many members of the community over many years. The initial version of this document was developed collaboratively through both the IAB IANA Strategy Program and the IETF IANAPLAN WG. Particular thanks go to Jari Arkko, Marc Blanchet, Brian Carpenter, Alissa Cooper, John Curran, Leslie Daigle, Heather Flanagan, Christer Holmberg, John Klensin, Barry Leiba, Milton Mueller, Andrei Robachevsky, Andrew Sullivan, Dave Thaler, Greg Wood, and Suzanne Woolf.

7. References

7.1. Normative References

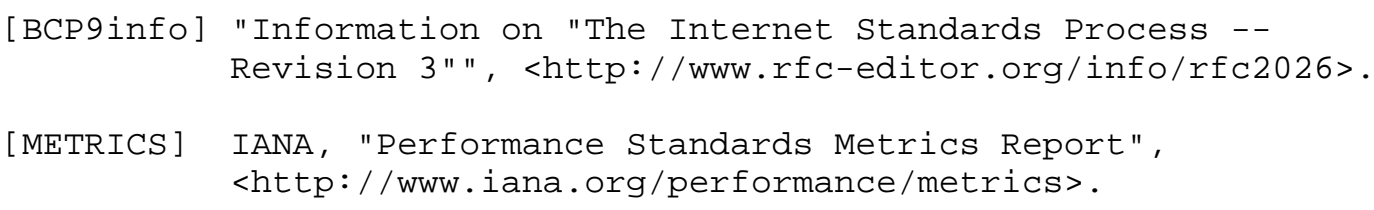


[MOUSUP] IAOC, "Supplements to RFC 2860 (the Memorandum of Understanding between the IETF and ICANN)", <http://iaoc.ietf.org/contracts.html>.

[NTIA-Announce ]

NTIA, "NTIA Announces Intent to Transition Key Internet Domain Name Functions", March 2014, <http://www.ntia.doc.gov/press-release/2014/ntiaannounces-intent-transition-key-internet-domain-namefunctions $>$.

[NTIA-Contract]

NTIA, "The NTIA Contract with ICANN", <http://www.ntia.doc.gov/files/ntia/publications/ sf_26_pg_1-2-final_award_and_sacs.pdf $>$.

[RFC2026] Bradner, S., "The Internet Standards Process -- Revision 3", BCP 9, RFC 2026, DOI 10.17487/RFC2026, October 1996, <http://www.rfc-editor.org/info/rfc2026>.

[RFC2418] Bradner, S., "IETF Working Group Guidelines and Procedures", BCP 25, RFC 2418, DOI 10.17487/RFC2418, September 1998, <http://wWw.rfC-editor.org/info/rfc2418>.

[RFC2850] Internet Architecture Board and B. Carpenter, Ed., "Charter of the Internet Architecture Board (IAB)", BCP 39, RFC 2850, DOI 10.17487/RFC2850, May 2000, <http://wWw.rfc-editor.org/info/rfc2850>.

[RFC2860] Carpenter, B., Baker, F., and M. Roberts, "Memorandum of Understanding Concerning the Technical Work of the Internet Assigned Numbers Authority", RFC 2860, DOI 10.17487/RFC2860, June 2000, <http://www.rfc-editor.org/info/rfc2860>.

[RFC3307] Haberman, B., "Allocation Guidelines for IPv6 Multicast Addresses", RFC 3307, DOI 10.17487/RFC3307, August 2002, <http://wWw.rfc-editor.org/info/rfc3307>.

[RFC3777] Galvin, J., Ed., "IAB and IESG Selection, Confirmation, and Recall Process: Operation of the Nominating and Recall Committees", RFC 3777, DOI 10.17487/RFC3777, June 2004, <http://www.rfc-editor.org/info/rfc3777>.

[RFC3935] Alvestrand, H., "A Mission Statement for the IETF", BCP 95, RFC 3935, DOI 10.17487/RFC3935, October 2004, <http://wWw.rfc-editor.org/info/rfc3935>. 
[RFC4071] Austein, R., Ed. and B. Wijnen, Ed., "Structure of the IETF Administrative Support Activity (IASA)", BCP 101, RFC 4071, DOI 10.17487/RFC4071, April 2005, <http://www.rfc-editor.org/info/rfc4071>.

[RFC5226] Narten, T. and H. Alvestrand, "Guidelines for Writing an IANA Considerations section in RFCs", BCP 26, RFC 5226, DOI 10.17487/RFC5226, May 2008, <http://www.rfc-editor.org/info/rfc5226>.

[RFC5771] Cotton, M., Vegoda, L., and D. Meyer, "IANA Guidelines for IPv4 Multicast Address Assignments", BCP 51, RFC 5771, DOI 10.17487/RFC5771, March 2010, <http://www.rfc-editor.org/info/rfc5771>.

[RFC6220] McPherson, D., Ed., Kolkman, O., Ed., Klensin, J., Ed., Huston, G., Ed., and Internet Architecture Board, "Defining the Role and Function of IETF Protocol Parameter Registry Operators", RFC 6220, DOI 10.17487/RFC6220, April 2011, <http://wWW.rfc-editor.org/info/rfc6220>.

[RFC6761] Cheshire, S. and M. Krochmal, "Special-Use Domain Names", RFC 6761, DOI 10.17487/RFC6761, February 2013, <http://www.rfc-editor.org/info/rfc6761>.

[RFC6890] Cotton, M., Vegoda, L., Bonica, R., Ed., and B. Haberman, "Special-Purpose IP Address Registries", BCP 153, RFC 6890, DOI 10.17487/RFC6890, April 2013, <http://www.rfc-editor.org/info/rfc6890>.

[RFC7282] Resnick, P., "On Consensus and Humming in the IETF", RFC 7282, DOI 10.17487/RFC7282, June 2014, <http://www.rfc-editor.org/info/rfc7282>.

7.2. Informative References

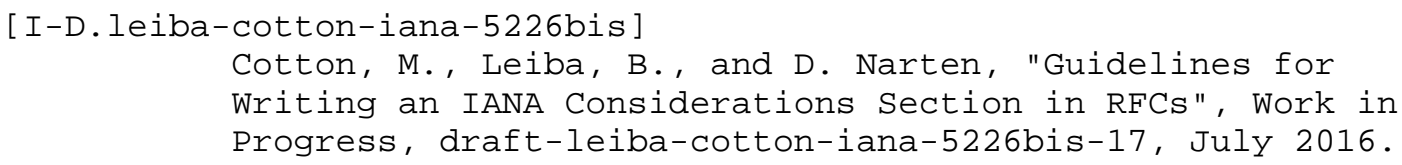


[ ICG-Response ]

IANA Stewardship Transition Coordination Group, "Proposal to Transition the Stewardship of the Internet Assigned Numbers Authority (IANA) Functions from the U.S. Commerce Department's National Telecommunications and Information Administration (NTIA) to the Global Multistakeholder Community", 11 March 2016, <https://www.icann.org/en/system/files/files/ iana-stewardship-transition-proposal-10mar16-en.pdf>.

[ProtoParamEvo14]

IAB Chair, "Subject: Re: [Internetgovtech] Guiding the Evolution of the IANA Protocol Parameter Registries", March 2014, <http://mailarchive.ietf.org/arch/msg/ internetgovtech/4EQ4bnEfE5ZkrPAtSAO2OBZM03k>.

[RFC-INDEX]

RFC Editor, "RFC Index",

<http://www.rfc-editor.org/rfc/rfc-index.txt>.

[RFC2014] Weinrib, A. and J. Postel, "IRTF Research Group Guidelines and Procedures", BCP 8, RFC 2014, DOI 10.17487/RFC2014, October 1996, <http://www.rfc-editor.org/info/rfc2014>.

[RFC2870] Bush, R., Karrenberg, D., Kosters, M., and R. Plzak, "Root Name Server Operational Requirements", RFC 2870, DOI 10.17487/RFC2870, June 2000, <http://www.rfc-editor.org/info/rfc2870>.

[RFC3172] Huston, G., Ed., "Management Guidelines \& Operational Requirements for the Address and Routing Parameter Area Domain ("arpa")", BCP 52, RFC 3172, DOI 10.17487/RFC3172, September 2001, <http://www.rfc-editor.org/info/rfc3172>.

[RFC4193] Hinden, R. and B. Haberman, "Unique Local IPv6 Unicast Addresses", RFC 4193, DOI 10.17487/RFC4193, October 2005, <http://www.rfc-editor.org/info/rfc4193>.

[RFC6793] Vohra, Q. and E. Chen, "BGP Support for Four-octet Autonomous System (AS) Number Space", RFC 6793, DOI 10.17487/RFC6793, December 2012, <http://www.rfc-editor.org/info/rfc6793>.

[RFC6852] Housley, R., Mills, S., Jaffe, J., Aboba, B., and L. St.Amour, "Affirmation of the Modern Paradigm for Standards", RFC 6852, DOI 10.17487/RFC6852, January 2013, <http://www.rfc-editor.org/info/rfc6852>. 
[RFC7020] Housley, R., Curran, J., Huston, G., and D. Conrad, "The Internet Numbers Registry System", RFC 7020, DOI 10.17487/RFC7020, August 2013, <http://www.rfc-editor.org/info/rfc7020>.

[RFC7249] Housley, R., "Internet Numbers Registries", RFC 7249, DOI 10.17487/RFC7249, May 2014, <http://www.rfc-editor.org/info/rfc7249>. 
Appendix A. The Charter of the IANA Stewardship Coordination Group ( ICG)

Charter for the IANA Stewardship Transition Coordination Group V.10

(August 27, 2014)

The IANA stewardship transition coordination group (ICG) has one deliverable: a proposal to the U.S. Commerce Department National Telecommunications and Information Administration (NTIA) regarding the transition of NTIA's stewardship of the IANA functions to the global multi-stakeholder community. The group will conduct itself transparently, consult with a broad range of stakeholders, and ensure that its proposals support the security and stability of the IANA functions.

The group's mission is to coordinate the development of a proposal among the communities affected by the IANA functions. The IANA functions are divided into three main categories: domain names, number resources, and other protocol parameters. The domain names category falls further into the country code and generic domain name sub-categories. While there is some overlap among all of these categories, each poses distinct organizational, operational and technical issues, and each tends to have distinct communities of interest and expertise. For those reasons it is best to have work on the three categories of IANA parameters proceed autonomously in parallel and be based in the respective communities.

The IANA stewardship transition process is taking place alongside a parallel and related process on enhancing ICANN accountability. While maintaining the accountability of Internet identifier governance is central to both processes, this group's scope is focused on the arrangements required for the continuance of IANA functions in an accountable and widely accepted manner after the expiry of the NTIA-ICANN contract. Nevertheless, the two processes are interrelated and interdependent and should appropriately coordinate their work.

The coordination group has four main tasks:

(i) Act as liaison to all interested parties, including the three "operational communities" (i.e., those with direct operational or service relationship with IANA; namely names, numbers, protocol parameters). This task consists of:

a. Soliciting proposals from the operational communities

b. Soliciting the input of the broad group of communities affected by the IANA functions

(ii) Assess the outputs of the three operational communities for compatibility and interoperability 
(iii) Assemble a complete proposal for the transition

(iv) Information sharing and public communication

Describing each in more detail:

(i) Liaison

a. Solicit proposals

The ICG expects a plan from the country code and generic name communities (possibly a joint one), a plan from the numbers community, and a plan from the protocol parameters community. Members of the ICG will ensure that the communities from which they are drawn are working on their part of the transition plans. This involves informing them of requirements and schedules, tracking progress, and highlighting the results or remaining issues. The role of a coordination group member during this phase is to provide status updates about the progress of his or her community in developing their component, and to coordinate which community will develop a transition proposal for each area of overlap (e.g., special-use registry).

While working on the development of their proposals, the operational communities are expected to address common requirements and issues relating to the transition, in as far as they affect their parts of the stewardship of IANA functions.

b. Solicit broader input

The ICG is open for input and feedback from all interested parties. While no set of formal requirements related to a transition proposal will be requested outside the operational communities, everyone's input is welcome across all topics.

The ICG expects that all interested parties get involved as early as possible in the relevant community processes. Input received directly by the ICG may be referred to the relevant community discussion.

The ICG members chosen from a particular community are the official communication channel between the ICG and that community.

\section{(ii) Assessment}

When the group receives output from the communities it will discuss and assess their compatibility and interoperability with the proposals of the other communities. Each proposal should be submitted with a clear record of how consensus has been reached for the proposal in the community, and provide an analysis that shows the 
proposal is in practice workable. The ICG should also compile the input it has received beyond the operational communities, and review the impacts of this input.

The ICG might at some point detect problems with the component proposals. At that point the role of the ICG is to communicate that back to the relevant communities so that they (the relevant communities) can address the issues. It is not in the role of the ICG to develop proposals or to select from among competing proposals.

(iii) Assembling and submitting a complete proposal

The assembly effort involves taking the proposals for the different components and verifying that the whole fulfills the intended scope, meets the intended criteria, that there are no missing parts, and that the whole fits together. The whole also needs to include sufficient independent accountability mechanisms for running the IANA function. The ICG will then develop a draft final proposal that achieves rough consensus within the ICG itself. The ICG will then put this proposal up for public comment involving a reasonable period of time for reviewing the draft proposal, analyzing and preparing supportive or critical comments. The ICG will then review these comments and determine whether modifications are required. If no modifications are needed, and the coordination group agrees, the proposal will be submitted to NTIA.

If changes are required to fix problems or to achieve broader support, the ICG will work with the operational communities in a manner similar to what was described in task (ii) above. Updates are subject to the same verification, review, and consensus processes as the initial proposals. If, in the ICG's opinion, broad public support for the proposal as articulated by the NTIA is not present, the parts of the proposal that are not supported return to the liaison phase.

(iv) Information sharing

The ICG serves as a central clearinghouse for public information about the IANA stewardship transition process. Its secretariat maintains an independent, publicly accessible and open website, under its own domain, where status updates, meetings and notices are announced, proposals are stored, the ICG members are listed, etc. As the development of the transition plans will take some time, it is important that information about ongoing work is distributed early and continuously. This will enable sharing of ideas and the detection of potential issues. 
Appendix B. IANA Stewardship Transition Coordination Group Request for Proposals

IANA Stewardship Transition Coordination Group Request for Proposals

8 September 2014

Introduction

Under the IANA Stewardship Transition Coordination Group (ICG)

Charter, the ICG has four main tasks:

(i) Act as liaison to all interested parties in the IANA stewardship transition, including the three "operational communities" (i.e., those with direct operational or service relationships with the IANA functions operator; namely names, numbers, protocol parameters). This task consists of:

a. Soliciting proposals from the operational communities

b. Soliciting the input of the broad group of communities affected by the IANA functions

(ii) Assess the outputs of the three operational communities for compatibility and interoperability

(iii) Assemble a complete proposal for the transition

(iv) Information sharing and public communication

This Request for Proposals (RFP) addresses task (i) of the ICG Charter. This RFP does not preclude any form of input from the non-operational communities.

\section{Complete Formal Responses}

The IANA Stewardship Transition Coordination Group (ICG) seeks complete formal responses to this RFP through processes which are to be convened by each of the "operational communities" of IANA (i.e., those with direct operational or service relationships with the IANA functions operator, in connection with names, numbers, or protocol parameters).

Proposals should be supported by the broad range of stakeholders participating in the proposal development process. Proposals should be developed through a transparent process that is open to and inclusive of all stakeholders interested in participating in the development of the proposal. In order to help the ICG maintain its light coordination role, all interested and affected parties are 
strongly encouraged to participate directly in these community processes.

The following link provides information about ongoing community processes and how to participate in them, and that will continue to be updated over time:

https://www.icann.org/en/stewardship/community

In this RFP, "IANA" refers to the functions currently specified in the agreement between NTIA and ICANN

[http://www.ntia.doc.gov/page/iana-functions-purchase-order] as well as any other functions traditionally performed by the IANA functions operator. SAC-067

[https://www.icann.org/en/system/files/files/sac-067-en.pdf] provides one description of the many different meanings of the term "IANA" and may be useful reading in addition to the documents constituting the agreement itself.

Communities are asked to adhere to open and inclusive processes in developing their responses, so that all community members may fully participate in and observe those processes. Communities are also asked to actively seek out and encourage wider participation by any other parties with interest in their response.

A major challenge of the ICG will be to identify and help to reconcile differences between submitted proposals, in order to produce a single plan for the transition of IANA

stewardship. Submitted Proposals should therefore focus on those elements that are considered to be truly essential to the transition of their specific IANA functions. The target deadline for all complete formal responses to this RFP is 15 January 2015.

\section{Comments}

While the ICG is requesting complete formal proposals through processes convened by each of the operational communities, and that all interested parties get involved as early as possible in the relevant community processes, some parties may choose to provide comments directly to the ICG about specific aspects of particular proposals, about the community processes, or about the ICG's own processes. Comments may be directly submitted to the ICG any time via email to icg-forumeicann.org. Comments will be publicly archived at <http://forum.icann.org/lists/icg-forum/>. 
Commenters should be aware that ICG will direct comments received to the relevant operational communities if appropriate. The ICG will review comments received as time and resources permit and in accordance with the overall timeline for the transition. That is, comments received about specific proposals may not be reviewed until those proposals have been submitted to the ICG. The ICG may establish defined public comment periods about specific topics in the future, after the complete formal responses to the RFP have been received.

Required Proposal Elements

The ICG encourages each community to submit a single proposal that contains the elements described in this section.

Communities are requested to describe the elements delineated in the sections below in as much detail possible, and according to the suggested format/structure, to allow the ICG to more easily assimilate the results. While each question is narrowly defined to allow for comparison between answers, respondents are encouraged to provide further information in explanatory sections, including descriptive summaries of policies/practices and associated references to source documents of specific policies/practices. In this way, the responses to the questionnaire will be useful at the operational level as well as to the broader stakeholder communities.

In the interest of completeness and consistency, proposals should cross-reference wherever appropriate the current IANA Functions Contract [3] when describing existing arrangements and proposing changes to existing arrangements.

0. Proposal type

Identify which category of the IANA functions this submission proposes to address:

[ ] Names [ ] Numbers [ ] Protocol Parameters

I. Description of Community's Use of IANA Functions

This section should list the specific, distinct IANA functions your community relies on. For each IANA function on which your community relies, please provide the following:

- A description of the function;

- A description of the customer(s) of the function;

o What registries are involved in providing the function; 
- A description of any overlaps or interdependencies between your IANA requirements and the functions required by other customer communities.

If your community relies on any other IANA service or activity beyond the scope of the IANA functions contract, you may describe them here. In this case please also describe how the service or activity should be addressed by the transition plan.

II. Existing, Pre-Transition Arrangements

This section should describe how existing IANA-related arrangements work, prior to the transition.

[3] http://www.ntia.doc.gov/files/ntia/ publications/sf_26_pg_1-2-final_award_and_sacs.pdf

A. Policy Sources

This section should identify the specific source(s) of policy which must be followed by the IANA functions operator in its conduct of the services or activities described above. If there are distinct sources of policy or policy development for different IANA functions, then please describe these separately. For each source of policy or policy development, please provide the following:

O Which IANA function (identified in section I) are affected.

- A description of how policy is developed and established and who is involved in policy development and establishment.

- A description of how disputes about policy are resolved.

- References to documentation of policy development and dispute resolution processes.

B. Oversight and Accountability

This section should describe all the ways in which oversight is conducted over the IANA functions operator's provision of the services and activities listed in section I and all the ways in which the IANA functions operator is currently held accountable for the provision of those services. For each oversight or accountability mechanism, please provide as many of the following as are applicable:

Which IANA functions (identified in Section I) are affected. If the policy sources identified in section II.A are affected, identify which ones are affected and explain in what way. 
- A description of the entity or entities that provide oversight or perform accountability functions, including how individuals are selected or removed from participation in those entities.

o A description of the mechanism (e.g., contract, reporting scheme, auditing scheme, etc.). This should include a description of the consequences of the IANA functions operator not meeting the standards established by the mechanism, the extent to which the output of the mechanism is transparent and the terms under which the mechanism may change.

o Jurisdiction(s) in which the mechanism applies and the legal basis on which the mechanism rests.

\section{Proposed Post-Transition Oversight and Accountability} Arrangements

This section should describe what changes your community is proposing to the arrangements listed in Section II.B in light of the transition. If your community is proposing to replace one or more existing arrangements with new arrangements, that replacement should be explained and all of the elements listed in Section II.B should be described for the new arrangements. Your community should provide its rationale and justification for the new arrangements.

If your community's proposal carries any implications for the interface between the IANA functions and existing policy arrangements described in Section II.A, those implications should be described here.

If your community is not proposing changes to arrangements listed in Section II.B, the rationale and justification for that choice should be provided here.

IV. Transition Implications

This section should describe what your community views as the implications of the changes it proposed in Section III. These implications may include some or all of the following, or other implications specific to your community:

Description of operational requirements to achieve continuity of service and possible new service integration throughout the transition.

Risks to operational continuity and how they will be addressed. Description of any legal framework requirements in the absence of the NTIA contract. Description of how you have tested or evaluated the workability of any new technical or operational methods proposed in this document and how they compare to established arrangements. 


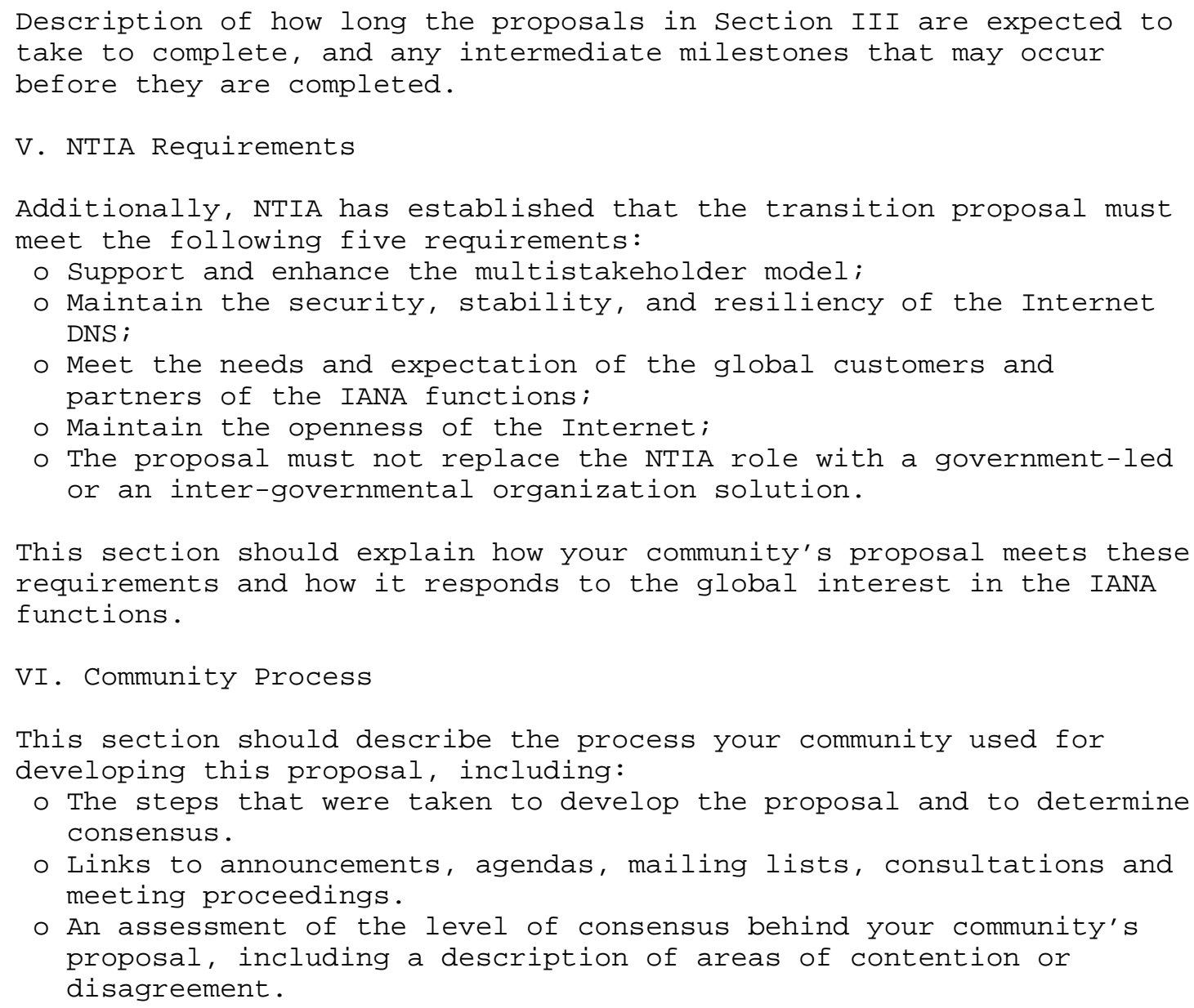


Appendix C. Correspondence of the IETF to the ICG

The following messages were sent to the ICG:

From: Jari Arkko <jari.arkko@piuha.net>

Subject: Re: [Internal-cg] Question from the ICG

Date: 20 Feb 2015 23:46:20 GMT+2

To: Alissa Cooper <alissa@cooperw.in>, ICG <internal-cg@icann.org>

Cc: Izumi Okutani <izumi@nic.ad.jp>

Dear Alissa and the ICG,

We refer to the question that the ICG asked the IETF community

on 9 Feb 2015

http://www.ietf.org/mail-archive/web/ianaplan/current/msg01610.html

$>$ The numbers proposal sees these changes as a requirement of the

$>$ transition and the protocols parameters proposal does not. If

$>$ these aspects of the proposals are perceived as incompatible would

$>$ the numbers and protocol parameters communities be willing to

$>$ modify their proposals to reconcile them?

We do not observe incompatibilities between the proposals from the numbers and protocol parameters communities. The numbers community expresses a preference to transfer the trademark and domain, while the IETF proposal does not oppose such transfer. This is not an incompatibility, it is something that can be satisfied by implementation of both number and protocol parameters community's proposals, as already specified.

To confirm this, and to determine whether the transfer

of the trademark and domain would be acceptable,

we consulted the community. It is the opinion of the

IANAPLAN working group that they would support a

decision by the IETF Trust to hold the trademark and domain

on behalf of the Internet community. For details, see

http://www. ietf.org/mail-archive/web/ianaplan/current/msg01659.html

The IETF Trust also looked at this issue. The trustees decided that the IETF Trust would be willing to hold intellectual property rights relating to the IANA function, including the IANA trademark and the IANA.ORG domain name. For details, see

http://www.ietf.org/mail-archive/web/ianaplan/current/msg01664.html

In short, we find no incompatibility between the proposals and no need to modify the protocol parameters proposal. 


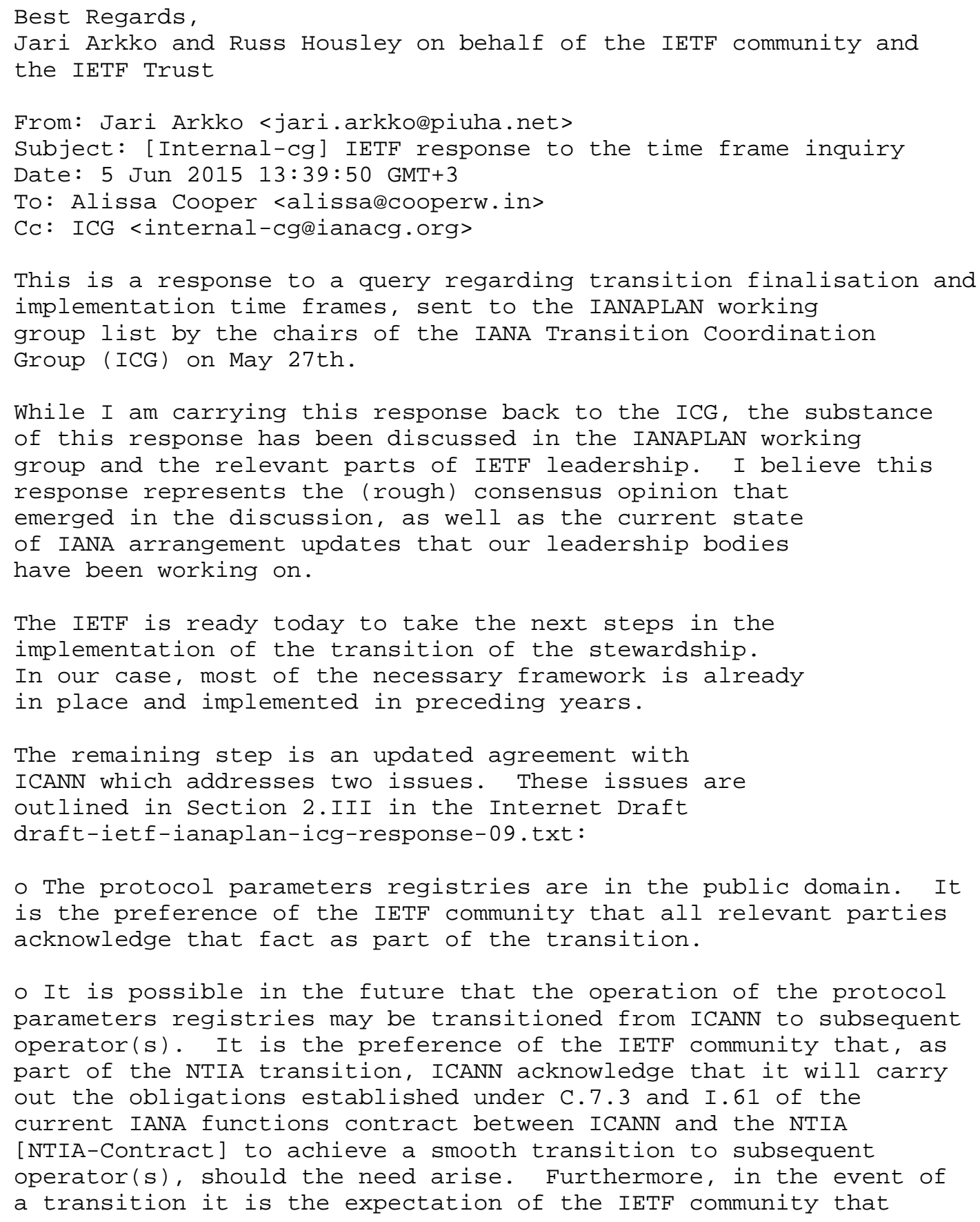


ICANN, the IETF, and subsequent operator(s) will work together to minimize disruption in the use of the protocol parameters registries or other resources currently located at iana.org.

The IETF Administrative Oversight Committee (IAOC) has decided to use an update of our yearly IETF-ICANN Service Level Agreement (SLA) as the mechanism for this updated agreement. They have drafted the update and from our perspective it could be immediately executed. Once the updated agreement is in place, the transition would be substantially complete, with only the NTIA contract lapse or termination as a final step.

Of course, we are not alone in this process. Interactions with other parts of the process may bring additional tasks that need to be executed either before or after the transition. First, the ICG, the RIRs, and IETF have discussed the possibility of aligning the treatment of IANA trademarks and domains. The IETF Trust has signalled that it would be willing to do this, if asked. We are awaiting coordination on this to complete, but see no problem in speedy execution once the decision is made. From our perspective this is not a prerequisite for the transition, however.

In addition, the names community has proposed the creation of a 'Post Transition IANA' (PTI). If the existing agreements between the IETF and ICANN remain in place and the SLAs discussed above are not affected, the IETF transition would take place as described above. That is our preference. If the final details of the PTI plan require further action from the IETF, more work and community agreement would be required. The timeline for that work cannot be set until the scope is known.

Jari Arkko, IETF Chair

(reporting his summary of the situation)

From: Jari Arkko <jari.arkko@piuha.net>

Subject: [Internal-cg] Response from IETF IANAPLAN WG regarding the ICG question on coordination

Date: 8 oct 2015 10:13:07 GMT+3

To: IANA etc etc Coordination Group <internal-cg@ianacg.org>

The IANAPLAN working group has discussed the coordination question from the ICG. In the working group's opinion, 
informal coordination exists today and will continue, which

is consistent with the commitment requested by the ICG.

This is also consistent with an overall coordination commitment already indicated in the IANAPLAN proposal. The proposal is a consensus document of the IETF. From the proposal:

The IETF will continue to coordinate with ICANN, the RIRs, and other parties that are mutually invested in the continued smooth operation of the Internet registries.

The coordination approach is also consistent with the comments that were sent by the IAB to the ICG during the public comment period. See https://www.iab.org/documents/correspondence-reports-documents/20152/iab-comments-on-icg-proposal/.

Jari Arkko,

IETF Chair and the Area Director for the IANAPLAN WG

Authors' Addresses

Eliot Lear (editor)

Richtistrasse 7

Wallisellen, ZH CH-8304

Switzerland

Phone: +41448789200

Email: lear@cisco.com

Russ Housley (editor)

918 Spring Knoll Drive

Herndon, VA 20170

United States of America

Email: housley@vigilsec.com 